\title{
Control of the Underactuated Inertia Wheel Inverted Pendulum for Stable Limit Cycle Generation
}

\author{
Sébastien Andary, Ahmed Chemori* and Sébastien Krut \\ LIRMM, University of Montpellier 2, CNRS, 161 rue Ada, 34392 Montpellier, France
}

Received 17 February 2009; accepted 3 July 2009

\begin{abstract}
This paper deals with a control approach dedicated to stable limit cycle generation for underactuated mechanical systems. The proposed approach is based on partial nonlinear feedback linearization and dynamic control for optimal periodic reference trajectories tracking. The computation of the reference trajectories is performed in order to optimize the behavior of the whole dynamics of the system and especially its zero dynamics at the end of each cycle. Simulation results as well as experiments show the performance and the efficiency of the proposed control scheme.
\end{abstract}

(C) Koninklijke Brill NV, Leiden and The Robotics Society of Japan, 2009

Keywords

Limit cycle, underactuated systems, optimization, partial feedback linearization, inertia wheel inverted pendulum

\section{Introduction}

Underactuated mechanical systems [1] are those systems that have less control inputs than generalized coordinates (degrees of freedom), i.e., they have generalized coordinates that are not actuated. In such systems the unactuated generalized coordinates may indirectly be controlled by the actuated coordinates through a dynamic coupling. This coupling is often nonlinear, hence the resulting dynamic constraints are generally nonintegrable and, therefore, second-order nonholonomic.

Many examples of such systems exist; mainly in robotics, they include, among others, the underactuated robot manipulators, the gymnast robots, particularly the acrobot [2], the pendubot [3], planar vertical takeoff and landing aircraft [4], undersea vehicles [5] and other mobile robots [6].

\footnotetext{
* To whom correspondence should be addressed. E-mail: chemori@lirmm.fr
} 
Underactuation in these mechanical systems is generally introduced intentionally at the design level to reduce the manufacturing cost, weight and/or failure rate, so the obtained systems may be able to perform complex tasks with a reduced number of actuators. However, such systems may require new approaches to design effective control strategies, therefore, they constitute a good framework of nonlinear control problems of both theoretical and practical interests. For these reasons they are attracting more and more attention from researchers from the nonlinear control community and robotics.

In the literature many research efforts have been made on control aspects $[2,3$, 7-11]; however, the field of control of such systems is still open to develop other control strategies.

In Ref. [9], a technique design combining partial feedback linearization and Lyapunov methods based on saturation functions, switching and energy shaping has been proposed. In Ref. [11], the author discusses a survey on some existing results such as geometric nonlinear control, and passivity based control for stabilizing and tracking control of such systems. Three control algorithms have been proposed in Ref. [8] for an underactuated two-link robot, i.e., an optimal LQ controller, a partial feedback linearizing regulator and a sliding mode controller.

In Ref. [3], the testbed is also a two-link underactuated robot, but the actuator is located at the base (in contrast to that proposed in Ref. [8], where the actuator is located at the joint between the two links) and the authors propose a method based on partial feedback linearization, nilpotent approximation and an iterative stabilization procedure. Roughly speaking they propose an open-loop controller that is able to steer the system closer to the desired equilibrium point in finite time and apply it iteratively, which provides a robust exponential convergence to the equilibrium.

In Ref. [10], the authors discuss the nonlinear behavior of the same robot proposed in Ref. [3] through a graphical tool based on the Poincare map. The study in Ref. [7] concerns underactuated mechanical systems with several actuated degrees of freedom and a single unactuated degree of freedom, where the authors propose a discontinuous nonlinear feedback controller that allows the closed-loop equilibrium at the origin to be globally attractive. In Ref. [12], a nonlinear predictive controller is proposed for both stabilization and stable limit cycle generation with an application to the ECP 505 inverted pendulum.

In this paper, a control approach based on collocated partial feedback linearization [11] and optimal reference trajectories is proposed, where the objective is the generation of stable limite cycles on both actuated and unactuated coordinates. The chosen application is the inertia wheel inverted pendulum. Simulation results show the effectiveness of the proposed control scheme; furthermore, experiments confirm the simulation results and show the robustness of the controller towards external disturbances.

This paper is organized as follows. In Section 2, the inertia inverted pendulum is described and its dynamic model is given. The generation of the optimal refer- 
ence trajectories as well as the control law are discussed in Section 3. Section 4 is dedicated to simulation results, while experiments are presented and discussed in Section 5. Finally, concluding remarks are drawn in Section 6.

\section{Inertia Wheel Inverted Pendulum}

The underactuated mechanical system studied in this paper is an inertia wheel inverted pendulum (Fig. 1) which consists of an inverted pendulum equipped with a rotating wheel. Its mechanical structure is sketched in Fig. 2. The motor torque produces an angular acceleration of the rotating wheel, which generates, thanks to the dynamic coupling, a torque acting on the pendulum's passive joint (as shown in Fig. 3); therefore, this passive joint can be controlled through the acceleration of the inertia wheel.

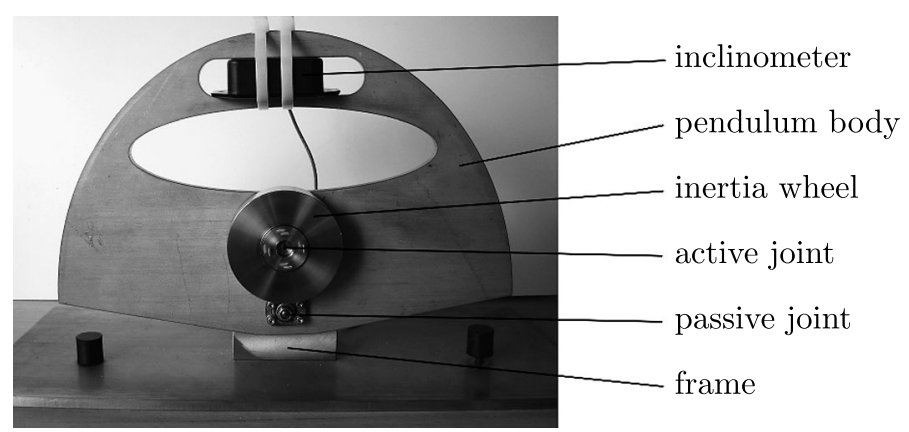

Figure 1. Inertia wheel inverted pendulum.

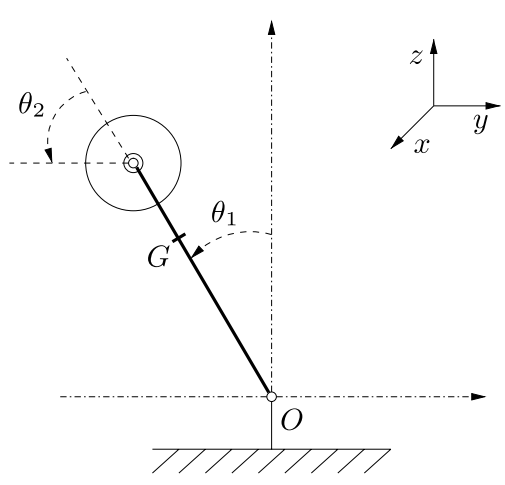

Figure 2. Schematic view of the system's mechanical structure: the joint between the frame and the pendulum body is unactuated (passive), while the joint between the body and the inertia wheel is actuated (active). 


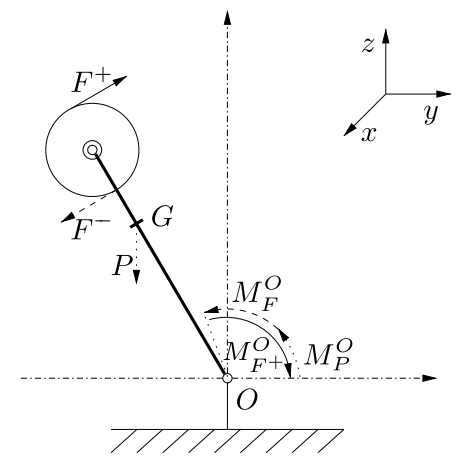

Figure 3. Mechanical principle: the inertia wheel is assumed to be equivalent to two punctual masses situated at the acting points of $F^{+}$and $F^{-}$. To straighten up the pendulum, the torque acting at $O$ generated by $F^{+}$must be greater than that generated by both gravity and $F^{-}$forces.

\subsection{Dynamic Modelling of the Plant}

The dynamic model of the system is obtained by application of the Lagrange formulation [13] and is described by:

$$
\left[\begin{array}{cc}
i_{1}+i_{2} & i_{2} \\
i_{2} & i_{2}
\end{array}\right]\left[\begin{array}{c}
\ddot{\theta}_{1} \\
\ddot{\theta}_{2}
\end{array}\right]+\left[\begin{array}{c}
-\overline{m l} g \sin \left(\theta_{1}\right) \\
0
\end{array}\right]=\left[\begin{array}{l}
C_{1} \\
C_{2}
\end{array}\right],
$$

where $\theta_{1}$ and $\theta_{2}$ are, respectively, angular positions of the pendulum body and the inertia wheel (Fig. 2), and $\dot{\theta}_{i}$ and $\ddot{\theta}_{i}, i=1, \ldots, 2$, represent their corresponding velocities and accelerations. $i_{1}$ and $i_{2}$ are, respectively, their moments of inertia. $C_{1}$ is the external disturbing torque applied to the pendulum while $C_{2}$ is the torque exerted by the actuator. $\overline{m l}=m_{1} l_{1}+m_{2} l_{2}$, where $m_{1}$ and $m_{2}$ are the masses of the pendulum and the inertia wheel, respectively, and $l_{1}$ and $l_{2}$ are distances from origin $O$ to the gravity center of the pendulum body and the inertia wheel, respectively. The proposed control approach is detailed in the next section.

\section{Proposed Control Scheme}

The objective of the control approach is to generate stable limit cycles on both actuated and unactuated coordinates. The system is underactuated since it has only one control input and 2 d.o.f. The control input can be used to track reference trajectories on only one coordinate. The question that may be asked is the following: which coordinate should be used to track the reference trajectories?

The choice of the coordinate on which the reference trajectories will be defined and tracked depends on the fixed objective. In our case, since the aim is to make the pendulum act like a metronome, the angle of the pendulum body $\theta_{1}$ has been chosen to be tracked.

The application of collocated partial feedback linearization allows us to linearize completely the dynamics of the unactuated coordinate $\theta_{1}$, whereas the remaining nonlinear dynamics of $\theta_{2}$ constitutes what is called the internal dynamics of the 
system. Since the system is nonminimum phase, the reference trajectories have to be optimized in order to ensure the stability of the internal dynamics. The optimization criterion is based on the rotation speed of the inertia wheel, which has to be minimized at the end of each cycle. Therefore, the idea is to propose parameterized reference trajectories and to compute their parameters by solving an optimization problem. The generation of the reference trajectories is discussed next.

\subsection{Reference Trajectories Generation}

Reference trajectories are to be established with the aim of generating stable limit cycles. Their shapes have to be chosen taking into account the following considerations:

(i) Since the desired dynamic behavior of the system is symmetrical with respect to the vertical, a periodic symmetrical reference trajectory should be chosen for $\theta_{1}$ in order to obtain stable periodic evolution on both actuated and unactuated coordinates. Indeed, a perfect tracking on $\theta_{1}$ of the nominal system leads to a periodic evolution of $\dot{\theta}_{2}$. Conversely, when the trajectory is asymmetrical with respect to the vertical, the pendulum rod will spend more time on one side and consequently the velocity of the inertia wheel at the end of the cycle will be different from zero.

(ii) The tracking of a symmetrical trajectory when the system is subject to uncertainties (e.g., on model parameters, external disturbances, etc.) leads to an aperiodic evolution of $\dot{\theta}_{2}$, i.e., its final value will be different from the initial value (zero value). For this reason, it will be clever to deform the original symmetrical reference trajectory in order to spend more time on one side to correct the asymmetrical evolution of $\dot{\theta}_{2}$. Doing that, the aim is to steer $\dot{\theta}_{2}$ to regain zero at the end of the cycle.

(iii) In our case, for smooth reference trajectories, periodic functions twice continuously differentiable are chosen to avoid discontinuities on velocities and accelerations.

Trajectories that fulfill the above considerations are designed as follows. They are split up in half-periods and each part is expressed by a parameterized polynomial function such that the reference trajectories on the whole cycle can be written as:

$$
\theta_{1}^{\mathrm{ref}}(t, p)= \begin{cases}A P\left(\frac{2 t}{\tau}, p\right) & \text { if } t \in\left[0, \frac{\tau}{2}[\right. \\ -A P\left(\frac{2 t}{\tau}-1,1-p\right) & \text { if } t \in\left[\frac{\tau}{2}, \tau\right],\end{cases}
$$

where $A$ and $\tau$ are, respectively, the amplitude and the period of oscillations, and $p$ is the optimization parameter. $P(t, p)$ is the normalized polynomial in $t$ that varies from 1 (at $t=0$ ) to -1 (at $t=1$ ) for all $p$. In order to join smoothly successive parts of the reference trajectories, the first and second time derivatives of 
$P(t, p)$ are constrained to be zero at boundaries ( $c f$. consideration (iii) above). Finally, $p$ is such that $P(t=p, p)=0$.

These considerations are mathematically expressed by the following constraints:

$$
\left\{\begin{array}{l}
P(0, p)=1 ; \quad P(t=p, p)=0 ; \quad P(1, p)=-1 \\
\frac{\partial P}{\partial t}(0, p)=0 ; \quad \frac{\partial P}{\partial t}(1, p)=0 \\
\frac{\partial^{2} P}{\partial t^{2}}(0, p)=0 ; \quad \frac{\partial^{2} P}{\partial t^{2}}(1, p)=0 .
\end{array}\right.
$$

Having seven constraints, then $P(t, p)$ can be defined as a six-degree $p$-parameterized polynomial in $t$, such that:

$$
P(t, p)=\sum_{i=0}^{6} \alpha_{i}(p) t^{i} .
$$

The identification of coefficients $\alpha_{i}(p)$ of the polynomial (4) taking into account constraint (iii) leads to the following:

$$
\begin{aligned}
& \alpha_{6}(p)=\frac{(2 p-1)\left(6 p^{4}-12 p^{3}+4 p^{2}+2 p+1\right)}{p^{3}(p-1)^{3}} \\
& \alpha_{5}(p)=\frac{-3\left(4 p^{6}-18 p^{4}+16 p^{3}-1\right)}{p^{3}(p-1)^{3}} \\
& \alpha_{4}(p)=\frac{3\left(10 p^{6}-18 p^{5}+10 p^{3}-1\right)}{p^{3}(p-1)^{3}} \\
& \alpha_{3}(p)=\frac{-\left(20 p^{6}-48 p^{5}+30 p^{4}-1\right)}{p^{3}(p-1)^{3}} \\
& \alpha_{2}(p)=0 \\
& \alpha_{1}(p)=0 \\
& \alpha_{0}(p)=1 .
\end{aligned}
$$

To show the effect of the parameter $p$ on the reference trajectory, Fig. 4 shows the evolution of the polynomial $P(t, p)$ for different values of $p$. Note that the pendulum spends the same amount of time on both sides of the equilibrium point when $p=0.5$.

For practical reasons (e.g., avoid touching mechanical stops) the passive joint $\theta_{1}$ of the pendulum is constrained to remain within interval $[-A, A]$; therefore, only a subinterval $\left[p_{\min }, p_{\max }\right]$ of $[0,1]$ is admissible for $p$ values. This requirement can be written for the normalized trajectories as:

$$
\forall p \in\left[p_{\min }, p_{\max }\right], \quad \forall t \in[0,1], \quad|P(t, p)| \leqslant 1,
$$

where $p_{\min }$ and $p_{\max }$ can be computed as follows. Assume for some $p$ that:

$$
\exists t_{1} \in[0,1] \text { s.t. }\left|P\left(t_{1}, p\right)\right|>1 .
$$




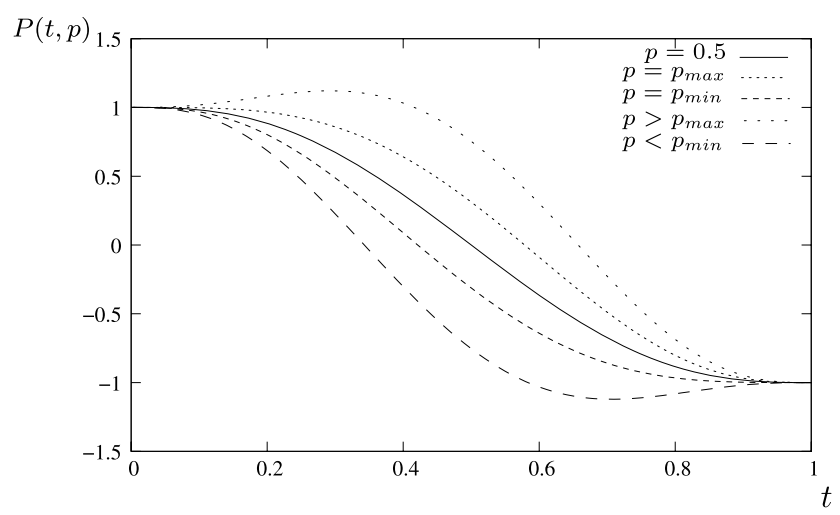

Figure 4. Evolution of the polynomial $P(t, p)$ for different values of $p$. Note that this corresponds to the normalized reference trajectory of $\theta_{1}$ during half a period.

Consider $P(0, p)=1, P(1, p)=-1$ and (6), $P$ must admit at least one null tangency point. It means that at least one root of $\partial P(t, p) / \partial t$ is within $[0,1]$. The computation of the roots of $\partial P(t, p) / \partial t$ leads to the following unique real solution:

$$
\frac{\partial P}{\partial t}\left(t_{1}, p\right)=0 \quad \Longrightarrow \quad t_{1}=\frac{20 p^{6}-48 p^{5}+30^{4}-1}{24 p^{5}-60 p^{4}+40 p^{3}-2} .
$$

The two extremal trajectories $P\left(t, p_{\min }\right)$ and $P\left(t, p_{\max }\right)$ correspond, respectively, to values 0 and 1 for $t_{1}$. This leads us to solve the following two equations:

$$
\begin{aligned}
& \frac{20 p^{6}-48 p^{5}+30 p^{4}-1}{24 p^{5}-60 p^{4}+40 p^{3}-2}=0 \\
& \frac{20 p^{6}-48 p^{5}+30 p^{4}-1}{24 p^{5}-60 p^{4}+40 p^{3}-2}=1 .
\end{aligned}
$$

Equation (8) admits a unique real solution $p=p_{\min }$, and (9) admits $p=p_{\max }$ as solution, both in interval $[0,1]$.

Remark 1. Numerical solution of (8) is $p_{\min } \simeq 0.42$, and that of (9) is $p_{\max } \simeq 0.58$, where it can be seen, due to the complementary feature between $p_{\min }$ and $p_{\max }$, that $p_{\min }+p_{\max }=1$.

\subsection{Tracking Control Law}

The dynamic model of the system, expressed by (1), can be rewritten as:

$$
\begin{gathered}
\ddot{\theta}_{1}=\frac{1}{i_{1}}\left(C_{1}-C_{2}+\overline{m l} g \sin \theta_{1}\right) \\
\ddot{\theta}_{2}=\frac{-1}{i_{1}} C_{1}+\frac{\left(i_{1}+i_{2}\right)}{i_{1} i_{2}} C_{2}+\frac{-\overline{m l} g}{i_{1}} \sin \theta_{1} .
\end{gathered}
$$


The above equations describe the system dynamics in open loop. According to collocated partial feedback linearization [9], consider the following control law (the torque $C_{1}$ is considered to be zero since the nominal system is not subject to external disturbing torque):

$$
C_{2}=\overline{m l} g \sin \left(\theta_{1}\right)-i_{1} \ddot{\theta}_{1}^{\text {ref }}+i_{1} k_{\mathrm{p}}\left(\theta_{1}-\theta_{1}^{\text {ref }}\right)+i_{1} k_{\mathrm{v}}\left(\dot{\theta}_{1}-\dot{\theta}_{1}^{\text {ref }}\right),
$$

where $k_{\mathrm{p}}$ and $k_{\mathrm{v}}$ are, respectively, the proportional and derivative feedback gains, and are used to tune the dynamics of the closed-loop system. Injecting this control law in (10) leads to the following linear closed-loop subsystem (completely linearized part):

$$
\ddot{e}+k_{\mathrm{v}} \dot{e}+k_{\mathrm{p}} e=0,
$$

where $e=\theta_{1}-\theta_{1}^{\text {ref }}, \dot{e}=\dot{\theta}_{1}-\dot{\theta}_{1}^{\text {ref }}$ and $\ddot{e}=\ddot{\theta}_{1}-\ddot{\theta}_{1}^{\text {ref }}$. By choosing the feedback gains $k_{\mathrm{p}}$ and $k_{\mathrm{v}}$ as positive definite matrices (scalar strictly positive gains in our case), the resulting linear system is asymptotically stable [14]. Therefore, $\lim _{t \rightarrow \infty} e=$ $\lim _{t \rightarrow \infty} \dot{e}=0$ and consequently $\theta_{1} \rightarrow \theta_{1}^{\text {ref }}$ and $\dot{\theta}_{1} \rightarrow \dot{\theta}_{1}^{\text {ref }}$ as $t \rightarrow \infty$. The pendulum passive joint then follows the reference trajectory $\theta_{1}^{\text {ref }}(t)$.

\subsection{Trajectory Optimization}

The aim of trajectory optimization is to minimize the motor rotating speed at the end of each cycle. In other words, for a given initial value $\dot{\theta}_{2}(0)$, the goal is to choose the optimal value of $p$ that minimizes a quadratic cost-function of $\dot{\theta}_{2}(\tau)$. Injecting the control law (12) in (10) and (11) leads to the following closed-loop system:

$$
\begin{gathered}
\ddot{\theta}_{1}=\ddot{\theta}_{1}^{\text {ref }}-k_{\mathrm{v}}\left(\dot{\theta}_{1}-\dot{\theta}_{1}^{\text {ref }}\right)-k_{\mathrm{p}}\left(\theta_{1}-\theta_{1}^{\text {ref }}\right) \\
\ddot{\theta}_{2}=-\frac{i_{1}+i_{2}}{i_{2}}\left(\ddot{\theta}_{1}^{\text {ref }}-k_{\mathrm{v}}\left(\dot{\theta}_{1}-\dot{\theta}_{1}^{\text {ref }}\right)-k_{\mathrm{p}}\left(\theta_{1}-\theta_{1}^{\text {ref }}\right)\right)+\frac{\overline{m l} g}{i_{2}} \sin \left(\theta_{1}\right) .
\end{gathered}
$$

Knowing the $p$-parametrized reference trajectory (2), numerical integration of (14) and (15), starting from initial condition $\left(\theta_{1}(0), \dot{\theta}_{1}(0), \dot{\theta}_{2}(0)\right)$, enables us to obtain the inertia wheel velocity at the end of the cycle $\dot{\theta}_{2}(\tau)$. Hence, the quadratic function to be minimized can be expressed by $g\left(p, \theta_{1}(0), \dot{\theta}_{1}(0), \dot{\theta}_{2}(0)\right)$. The optimization problem can then be formulated as:

$$
\hat{p}=\operatorname{Arg} \min _{p} g\left(p, \theta_{1}(0), \dot{\theta}_{1}(0), \dot{\theta}_{2}(0)\right) .
$$

This problem is to be solved at the beginning of each cycle. Its solution allows us to define completely the reference trajectory to be tracked on the next oscillation cycle.

\section{Simulation Results}

Consider the dynamic model of system (1) with dynamical parameters described in Table 1. These parameters have been identified thanks to the real prototype of the system. 
Table 1.

Description of dynamical parameters of the inverted pendulum

\begin{tabular}{llll}
\hline Parameter & Description & Value & Unit \\
\hline$m_{1}$ & Body mass & 3.228 & $\mathrm{~kg}$ \\
$m_{2}$ & Wheel mass & 0.86422 & $\mathrm{~kg}$ \\
$i_{1}$ & Body inertia & $3.042 \times 10^{-2}$ & $\mathrm{kgm}^{2}$ \\
$i_{2}$ & Wheel inertia & $7.986 \times 10^{-4}$ & $\mathrm{kgm}^{2}$ \\
$l_{1}$ & Body center of mass position & $6.354 \times 10^{-4}$ & $\mathrm{~m}$ \\
$l_{2}$ & Wheel center of mass position & $52 \times 10^{-3}$ & $\mathrm{~m}$ \\
\hline
\end{tabular}

Table 2.

Description of the reference trajectories and control parameters

\begin{tabular}{llcl}
\hline Parameter & Description & Value & Unit \\
\hline$A$ & Oscillation amplitude & 3 & $\mathrm{deg}$ \\
$\tau$ & Oscillation period & 2 & $\mathrm{~s}$ \\
$k_{\mathrm{p}}$ & Proportional gain & 300 & $1 / \mathrm{s}^{2}$ \\
$k_{\mathrm{V}}$ & Derivative gain & 20 & $1 / \mathrm{s}$ \\
\hline
\end{tabular}

Simulation results, obtained using Matlab software, are presented and discussed in the following. They attest to the feasibility of the proposed control scheme. Table 2 lists the parameters of the reference trajectories and the control approach.

The proposed simulation is started from the initial condition $\left(\theta_{1}(0), \dot{\theta}_{1}(0)\right.$, $\left.\dot{\theta}_{2}(0)\right)=\left(10^{\circ}, 0 \mathrm{rad} / \mathrm{s}, 0 \mathrm{rad} / \mathrm{s}\right)$. The choice of $\theta_{1}(0)$ is motivated by the physical properties of the inverted pendulum (pendulum angle value is approximatively $\pm 10^{\circ}$ at standstill) since simulation is started from rest.

Figure 5 displays the whole simulation results. Figure $5 \mathrm{a}$ and $5 \mathrm{~b}$ shows the pendulum body joint position and velocity versus time in solid lines, while the dashed lines represent their corresponding reference position and velocity. This shows clearly the convergence of position and velocity to their reference trajectories. The inertia wheel velocity versus time is displayed in Fig. $5 \mathrm{c}$ where it can be observed that within two periods, the motor velocity reaches a limit cycle and oscillates around zero. Figure 5e represents the control input that consists of the motor driver voltage (proportional to the motor torque), where it can be noticed that it remains within the admissible limits $( \pm 10 \mathrm{~V})$.

The phase portrait $\left(\theta_{1}, \dot{\theta}_{1}\right)$ is depicted in Fig. 5d, showing clearly the convergence from the initial condition to a stable limit cycle. Figure $5 f$ represents the absolute value of motor velocity versus absolute motor torque and the admissible region of the actuator, given by the manufacturer of the DC motor; it can be seen that the trajectories remain inside the admissible region. Finally, in Fig. 5g, the evo- 
(a) Angular position of the pendulum rod

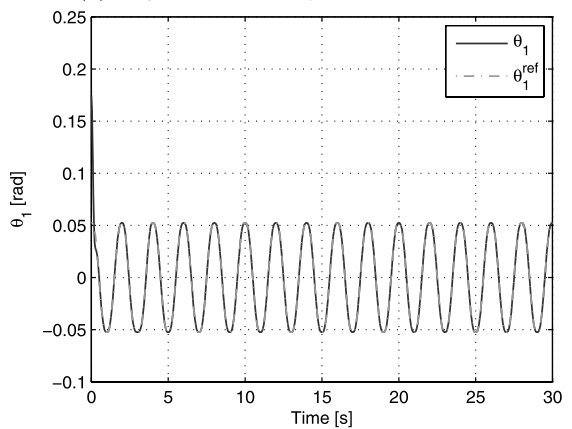

(c) Inertia wheel rotation speed

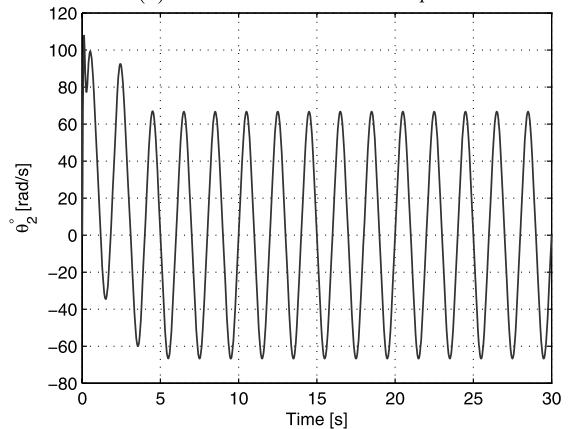

(e) Control input

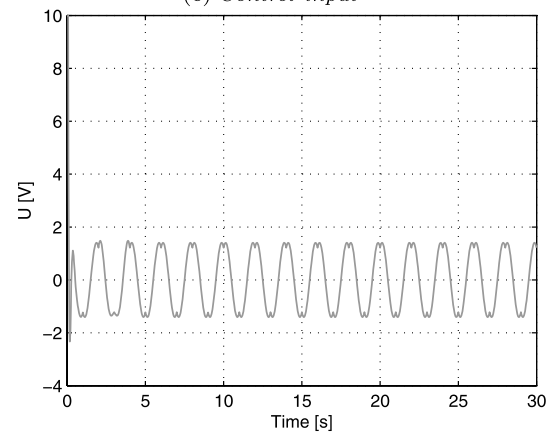

(b) Angular velocity of the pendulum rod

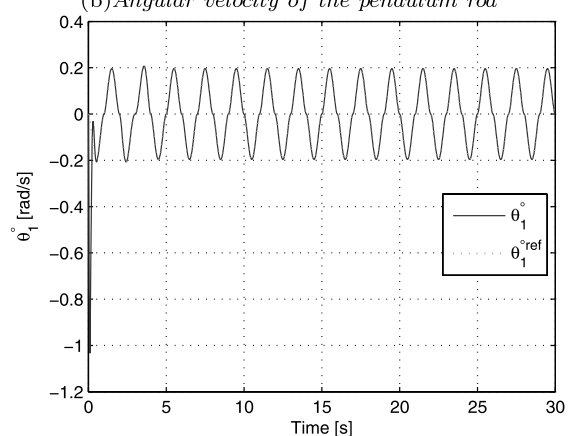

(d) Phase portrait $\left(\theta_{1}, \dot{\theta}_{1}\right)$

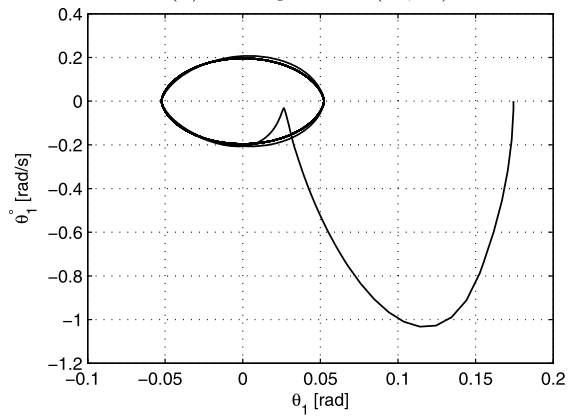

(f) Check of power admissible-region of the actuator

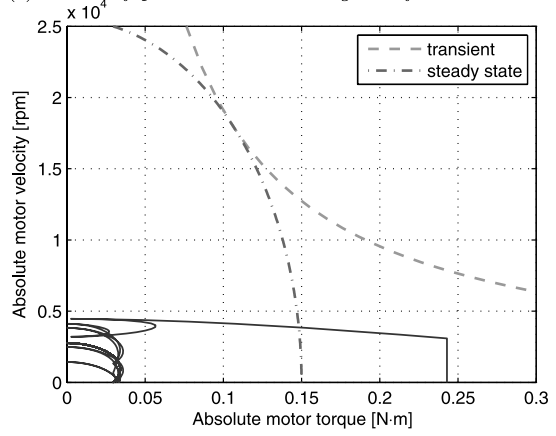

(g) Optimization parameter

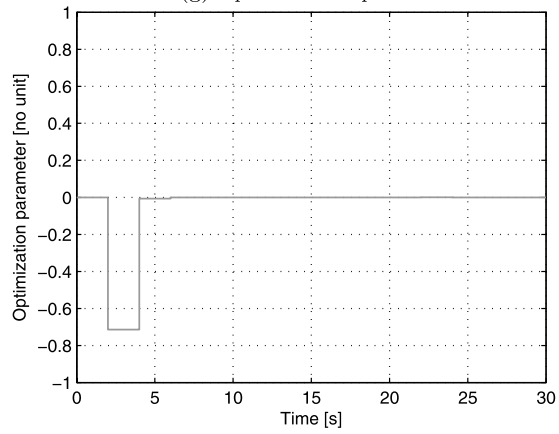

Figure 5. Simulation results with $A=3^{\circ}, \tau=2 \mathrm{~s}$. 
lution of the normalized optimal parameter (which evolves within interval $[-1,1]$ ) is displayed, where it converges after two cycles to a null value.

\section{Experimental Results}

In this section, the experimental results are represented with implementation issues. It starts with a description of the inverted pendulum testbed, and then two experiments are presented. The first one concerns the control of the disturbance-free real system, whereas in the second one the system is subject to external perturbations.

\subsection{Testbed and Implementation Issues}

Real-time experiments are performed on an inertia wheel inverted pendulum (Fig. 1) designed and developed at our laboratory. The pendulum angle $\theta_{1}$ is constrained to remain within the interval $\left[-10^{\circ}, 10^{\circ}\right]$ due to mechanical stops. The actuator of the plant is a Maxon EC-powermax 30 DC motor, equipped with an incremental encoder, allowing us to measure the inertia wheel angular position and to compute its velocity in real-time. In order to measure the angle of the pendulum with respect to the vertical, the system is equipped with a Micro strain FAS-G inclinometer. The choice of this solution, other than a classical encoder, has been made since it does not require us to set the frame horizontally. The system is controlled with a computer equipped with a 2.4-GHz Intel processor. The control approach is implemented using $\mathrm{C}$ language and the whole system runs under the Ardence RTX real-time OS.

The proposed control scheme requires that an optimization problem be solved in real-time; however, to overcome the problem of computation time, online implementation is performed using a lookup table. For that a grid is defined for different initial values $\dot{\theta}_{2}(0)$. Since the reference trajectories are periodic and the proposed controller is performant for tracking them, the influence of $\theta_{1}(0)$ and $\dot{\theta}_{1}(0)$ on the optimization parameter $p$ is minor. For that reason, the lookup table was defined only on $\dot{\theta}_{2}(0)$.

The optimization parameter (16) is computed offline for each value of $\dot{\theta}_{2}(0)$ on the grid and stored in a table. Then, real-time implementation of the proposed control scheme uses this lookup table as well as an interpolation technique to obtain instantaneously the optimization parameter.

\subsection{Real-Time Experiments}

In this section, real-time experiments are presented and discussed. They are performed on the inverted pendulum testbed presented in the previous section. Two experimental scenarios are presented and discussed. In the first one the proposed control scheme is implemented without considering any external disturbance, whereas in the second one, to show the robustness of the proposed approach, the system is subject to external disturbances. 


\subsubsection{Scenario 1: Without External Disturbances}

Figure 6 shows the whole results of this scenario. The pendulum joint position and velocity are displayed in Fig. 6a and 6b, respectively. Noise can be observed on the pendulum body velocity $\dot{\theta}_{1}$ since this last one is computed using a numerical derivation of the measured angular position $\theta_{1}$.

Figure $6 \mathrm{c}$ represents motor velocity versus time and shows how the optimization of the reference trajectories brings the motor velocity oscillation around zero in a few periods. DC motor input voltage can be observed in Fig. 6e where it is shown that it remains within the admissible range $\pm 10 \mathrm{~V}$. The phase portrait $\left(\theta_{1}, \dot{\theta}_{1}\right)$ is displayed in Fig. 6d. The limit cycle is reached even in the presence of noise. Figure $6 f$ shows the admissible region of the motor power. Finally, Fig. $6 \mathrm{~g}$ represents the evolution of the normalized optimization parameter.

This experiment is carried out with nonzero initial conditions; however, despite this fact the controller is able to bring the system to the reference trajectory while bringing back and keeping the inertia wheel angular velocity oscillation around zero.

\subsubsection{Scenario 2: With External Disturbances}

In this scenario, disturbances are introduced by pushing the pendulum, which generates external punctual torques applied to the pendulum joint at approximately $t=16$ and $33 \mathrm{~s}$.

Figure 7 represents the whole experimental results obtained with this second scenario. In Fig. 7a, the pendulum joint position and its corresponding reference trajectory are plotted versus time. Figure $7 \mathrm{~b}$ displays the curve of the pendulum joint velocity as well as its reference trajectory. On this curve, the effect of external disturbances can be observed as peaks that appear at instants of application of the external disturbing torques.

External disturbance compensation can be observed in Fig. 7c and 7e where, respectively, inertia wheel velocity and motor input voltage are displayed. One clearly observes peaks at the instant of disturbance and how the system brings back the motor velocity oscillation around zero after each peak.

The phase portrait and the motor's admissible power are displayed in Fig. 7d and $7 \mathrm{f}$, respectively. The limit cycle is rejoined after each perturbation. Figure $7 \mathrm{~g}$ represents the evolution of the obtained normalized optimization parameter versus time.

The controller is able to keep the system around the reference trajectories and reject external disturbances introduced as unpredicted torques applied on the pendulum axis in a punctual fashion.

\section{Conclusions and Future Work}

In this paper, a control approach is proposed for generating stable limit cycles for underactuated mechanical systems. The control scheme is designed in the special case of the inertia wheel inverted pendulum; however, it can be easily generalized 
(a) Angular position of the pendulum rod

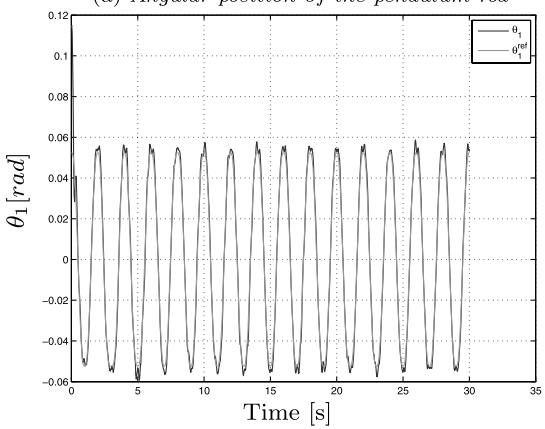

(c) Inertia wheel rotation speed

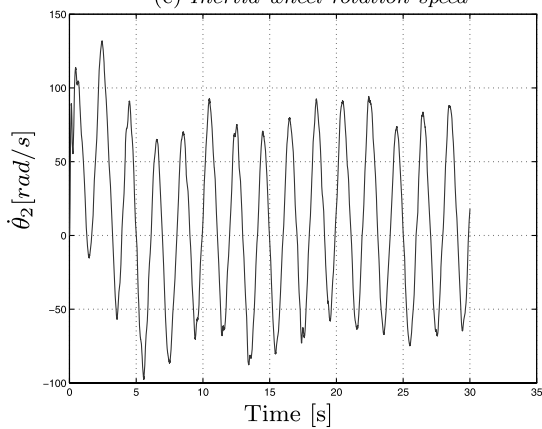

(e) Control input

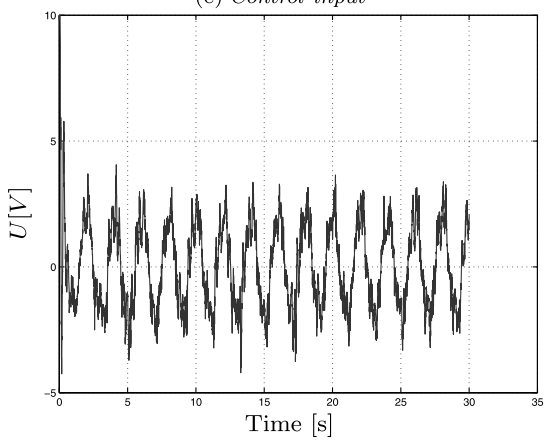

(b) Angular velocity of the pendulum rod

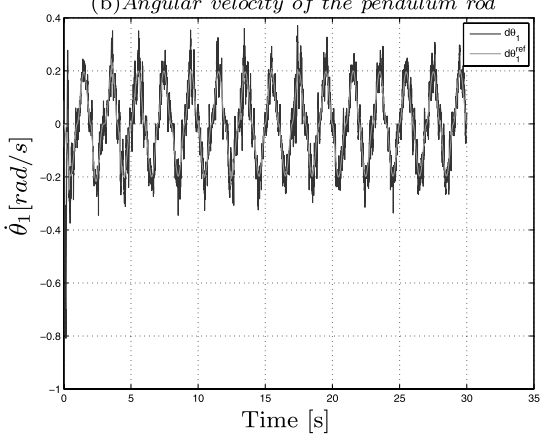

(d) Phase portrait $\left(\theta_{1}, \dot{\theta}_{1}\right)$

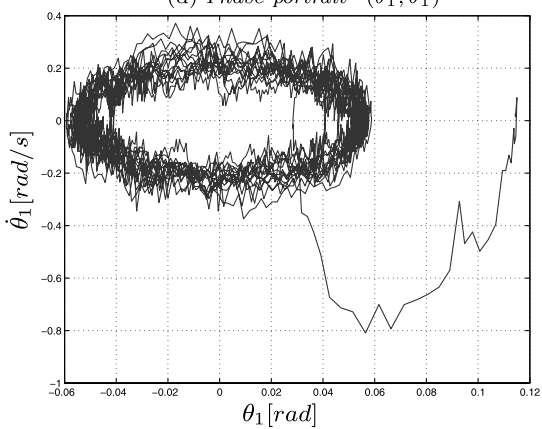

(f) Check of power admissible-region of the actuator

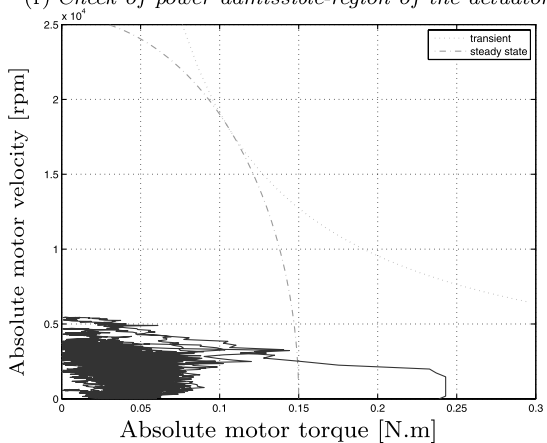

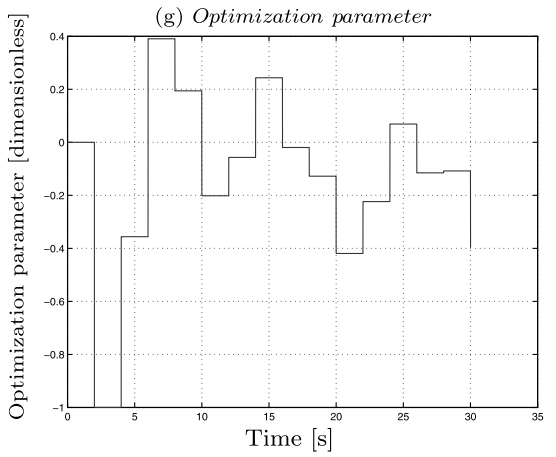

Figure 6. Experimental results with $A=3^{\circ}, \tau=2 \mathrm{~s}$ (without external disturbances). 
(a) Angular position of the pendulum rod

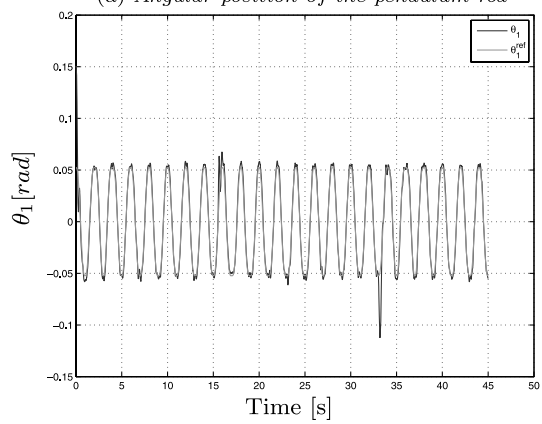

(c) Inertia wheel rotation speed

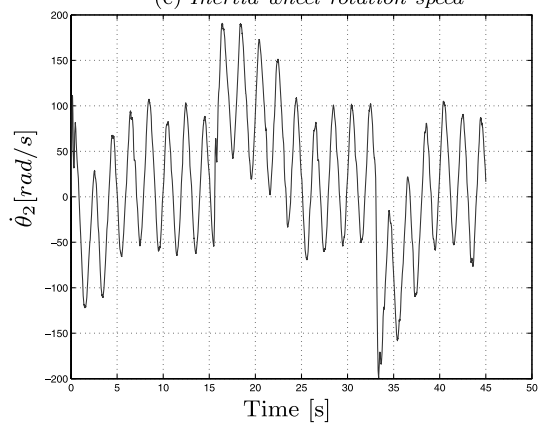

(e) Control input

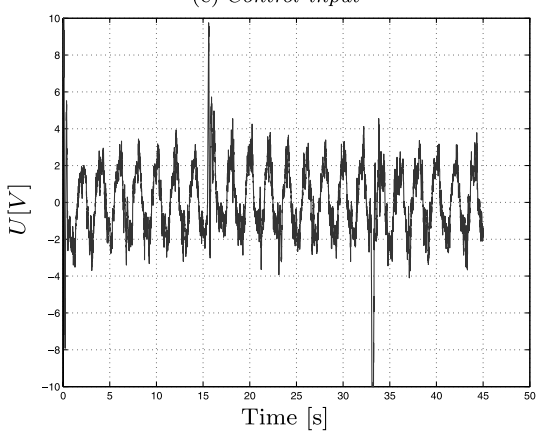

(b) Angular velocity of the pendulum rod

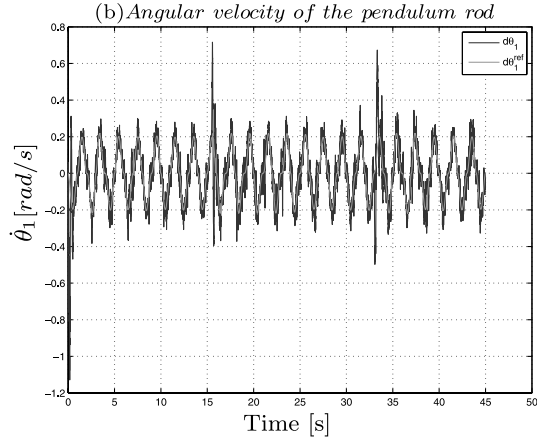

(d) Phase portrait $\left(\theta_{1}, \dot{\theta}_{1}\right)$

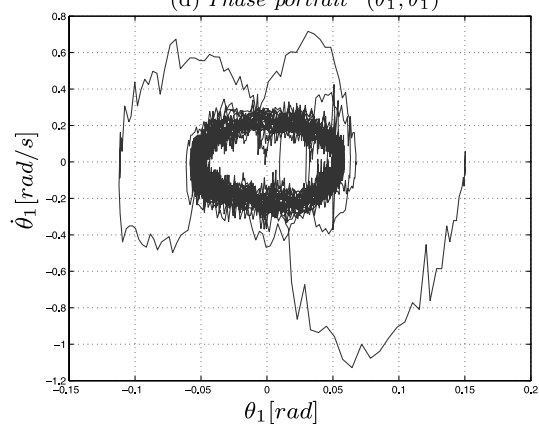

(f) Check of power admissible-region of the actuator

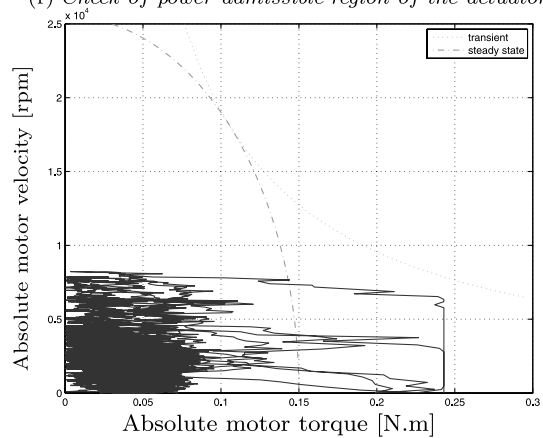

(g) Optimization parameter

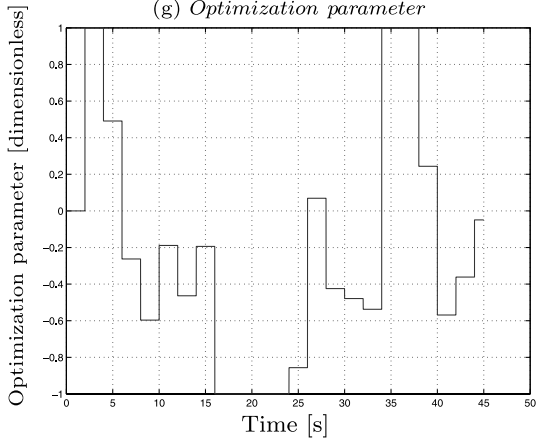

Figure 7. Experimental results with $A=3^{\circ}, \tau=2 \mathrm{~s}$ (with external disturbances). 
to the more general case of underactuated mechanical systems. It is based on collocated partial feedback linearization and optimal reference trajectories tracking. The obtained results in simulation and real-time experiments are presented and discussed to show the effectiveness and the performance of the proposed scheme.

Currently, the work is ongoing trying to find an analytical solution to the optimization problem. Furthermore, the robustness of the control scheme can be significantly improved by adopting more flexible reference trajectories and updating them many times during a cycle (in the actual version of the controller this is done only once a period).

\section{References}

1. I. Fantoni and R. Lozano, Non-linear Control for Underactuated Mechanical Systems. Springer, New York, NY (2001).

2. M. Spong, The swing up control problem for the acrobot, IEEE Control Syst. Mag. 15, 49-55 (1995).

3. A. De Luca, R. Mattone and G. Oriolo, Stabilization of underactuated planar $2 r$ manipulator, Int. J. Robust Nonlinear Control 10, 181-198 (2000).

4. G. Poulin, A. Chemori and N. Marchand, Minimum energy oriented global stabilizing control of the PVTOL aircraft, Int. J. Control 80, 430-442 (2007).

5. T. I. Fossen, Nonlinear modelling and control of underwater vehicles, PhD Thesis, Norwegian Institute of Technology (1991).

6. S. Ge and F. Lewis, Autonomous Mobile Robots. CRC Press, New York, NY (2006).

7. M. Reyhanoglu, S. Ludvigsen and O. Egeland, Discontinuous feedback control of a special class of underactuated mechanical systems, Int. J. Robust Nonlinear Control 10, 265-282 (2000).

8. K. Lee and V. Coverstone-Carroll, Control algorithms for stabilizing underactuated robots, J. Robotic Syst. 15, 681-697 (1998).

9. M. Spong, Energy based control of a class of underactuated mechanical system, in: Proc. 13th IFAC World Congr., San Francisco, CA, pp. 431-436 (1996).

10. Y. Nakamura, T. Suzuki and M. Koinuma, Nonlinear behavior and control of nonholonomic freejoint manipulator, IEEE Trans. Robotics Automat. 13, 853-862 (1997).

11. M. Spong, Underactuated mechanical systems, in: Control Problems in Robotics and Automation, B. Siciliano and K. P. Valavanis (Eds), pp. 135-150. Springer, New York, NY (1998).

12. A. Chemori and M. Alamir, Nonlinear predictive control of under-actuated mechanical systems, application: the ECP 505 inverted pendulum, in: Proc. Mathematical Theory of Networks and Systems, Leuven (2004).

13. M. Spong and M. Vidyasagar, Robot Dynamics and Control. Wiley, New York, NY (1989).

14. H. Khalil. Nonlinear Systems, 2nd edn. Prentice Hall, Upper Saddle River, NJ (1996). 


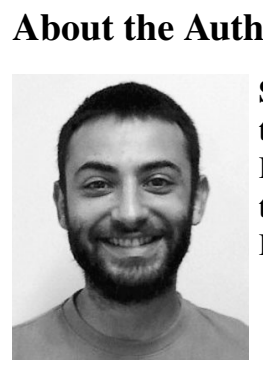

Sébastien Andary received the BS and MS degrees in Computer Science from the University of Sciences, Montpellier, France, in 2004 and 2006, respectively. $\mathrm{He}$ is now a PhD student at the University of Sciences, Montpellier, working for the Montpellier Laboratory of Computer Science, Microelectronics and Robotics. His research interests include robotics, nonlinear control and artificial learning.

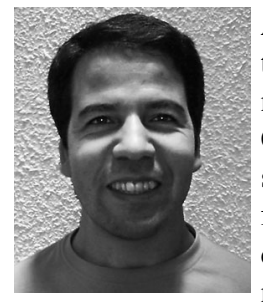

Ahmed Chemori received the MS degree in Automatic Control, in 2001, and the $\mathrm{PhD}$ degree in Automatic Control, in 2005, from Institut National Polytechnique de Grenoble, France. He was a Post-doctoral fellow with the Automatic Control Laboratory of Grenoble, France, in 2006. He is currently a tenured Research Scientist in Automatic Control and Robotics for the French National Center for Scientific Research, at the Montpellier Laboratory of Computer Science, Microelectronics and Robotics, Montpellier, France. His research interests include nonlinear control, adaptive control, predictive control and their applications in robotics.

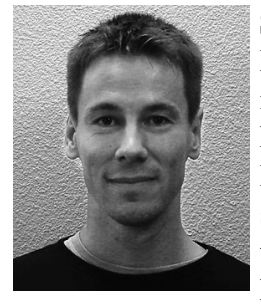

Sébastien Krut received the MS degree in Mechanical Engineering from the Pierre and Marie Curie University, Paris, France, in 2000, and the $\mathrm{PhD}$ degree in Automatic Control from the Montpellier University of Sciences, Montpellier, France, in 2003. He was a Post-doctoral fellow with the Joint Japanese-French Robotics Laboratory, Tsukuba, Japan, in 2004. He is currently a tenured research scientist in Robotics for the French National Center for Scientific Research, at the Montpellier Laboratory of Computer Science, Microelectronics and Robotics, Montpellier, France. His research interests include design and control of robotic systems. 